\title{
An Upper Limit on the Reflected Light from the Planet Orbiting the Star T Bootis
}

\section{Citation}

Charbonneau, David, Robert W. Noyes, Sylvain G. Korzennik, Peter Nisenson, Saurabh Jha, Steven S. Vogt, and Robert I. Kibrick. 1999. "An Upper Limit on the Reflected Light from the Planet Orbiting the Star t Bootis." The Astrophysical Journal 522 (2): L145-48. https:// doi.org/10.1086/312234.

\section{Permanent link}

http://nrs.harvard.edu/urn-3:HUL.InstRepos:41397430

\section{Terms of Use}

This article was downloaded from Harvard University's DASH repository, and is made available under the terms and conditions applicable to Other Posted Material, as set forth at http:// nrs.harvard.edu/urn-3:HUL.InstRepos:dash.current.terms-of-use\#LAA

\section{Share Your Story}

The Harvard community has made this article openly available.

Please share how this access benefits you. Submit a story.

Accessibility 
The Astrophysical Journal, 522:L145-L148, 1999 September 10

(C) 1999. The American Astronomical Society. All rights reserved. Printed in U.S.A.

\title{
AN UPPER LIMIT ON THE REFLECTED LIGHT FROM THE PLANET ORBITING THE STAR $\tau$ BOOTIS
}

\author{
David Charbonneau, Robert W. Noyes, Sylvain G. Korzennik, Peter Nisenson, and Saurabh Jha \\ Harvard-Smithsonian Center for Astrophysics, 60 Garden Street, Cambridge, MA 02138; dcharbonneau@cfa.harvard.edu \\ AND \\ Steven S. Vogt and Robert I. Kibrick \\ UCO/Lick Observatory, University of California at Santa Cruz, Santa Cruz, CA 95064 \\ Received 1999 May 26; accepted 1999 July 12; published 1999 August 13
}

\begin{abstract}
The planet orbiting $\tau$ Boo at a separation of 0.046 AU could produce a reflected light flux as bright as $1 \times$ $10^{-4}$ relative to that of the star. A spectrum of the system will contain a reflected light component which varies in amplitude and Doppler shift as the planet orbits the star. Assuming the secondary spectrum is primarily the reflected stellar spectrum, we can limit the relative reflected light flux to be less than $5 \times 10^{-5}$. This implies an upper limit of 0.3 for the planetary geometric albedo near $480 \mathrm{~nm}$, assuming a planetary radius of $1.2 R_{\text {Jup }}$. This albedo is significantly less than that of any of the giant planets of the solar system and is not consistent with certain published theoretical predictions.
\end{abstract}

Subject headings: planetary systems — stars: individual ( $\tau$ Bootis) — techniques: spectroscopic

\section{INTRODUCTION}

Radial velocity surveys of nearby F, G, K, and M dwarf stars have revealed eight planets (Mayor \& Queloz 1995; Butler et al. 1997; Butler et al. 1998; Fischer et al. 1999) ${ }^{1}$ which orbit their parent stars with a separation of $a \lesssim 0.1$ AU. These closein extrasolar giant planets (CEGPs) may be directly detectable by their reflected light, due to the proximity of the planet to the illuminating star. In this Letter, we present the results of a spectroscopic search for the reflected light component from the planet orbiting the star $\tau$ Boo. The motivation to attempt such a detection for a CEGP is strong: it would constitute the first direct detection of a planet orbiting another star. It would yield the orbital inclination, and hence the planetary mass, and would also measure a combination of the planetary radius and albedo, from which a minimum radius can be deduced. Furthermore, it would open the way to direct investigation of the spectrum of the planet itself. Conversely, a low enough upper limit would provide useful constraints on the radius and albedo of the CEGP.

\section{REFLECTED LIGHT}

\subsection{Photometric Variations}

In order to calculate the predicted flux ratio of the planet relative to the star, let $R_{p}$ denote the planetary radius, $R_{s}$ the stellar radius, $a$ the physical separation, and $\alpha$ the angle between the star and the observer as seen from the planet. The observationally useful quantity is the geometric albedo $p$, which is the flux from the planet at $\alpha=0$ divided by the flux that would be measured from a Lambert law (i.e., perfectly diffusing; see, e.g., Sobolev 1975) disk of the same diameter, located at the distance of the planet. In the case that $R_{p} \ll R_{s} \ll a$, the ratio $\epsilon$ of the observed flux from the planet at $\alpha=0$ to that of the

${ }^{1}$ See http://obswww.unige.ch/ $/$ udry/planet/hd75289_text.html, by M. Mayor, D. Naef, S. Udry, N. Santos, D. Queloz, C. Melo, \& B. Confino, and ESO press release 18/98 by D. Queloz et al. (http://www.eso.org/outreach/ press-rel/pr-1998/pr-18-98.html). star is

$$
\epsilon=p\left(\frac{R_{p}}{a}\right)^{2}
$$

The value of $p$ depends on the amplitude and angular dependence of the various sources of scattering in the planetary atmosphere, integrated over the surface of the sphere. For a Lambert law sphere, $p=2 / 3$, whereas for a semi-infinite purely Rayleigh scattering atmosphere, $p=3 / 4$. The geometric albedos at $480 \mathrm{~nm}$ of Jupiter, Saturn, Uranus, and Neptune are $0.46,0.39,0.60$, and 0.58, respectively (Karkoschka 1994).

We treat the orbit as circular, since the observed orbit of $\tau$ Boo has an eccentricity less than 0.02 (Butler et al. 1997). We neglect occultations, since a transit would produce a $\sim 0.01$ mag photometric dip and is ruled out by Baliunas et al. (1997). We take the orbital phase $\Phi \in[0,1]$ to be 0 at the time of maximum radial velocity of the star. The phase angle $\alpha \in[0, \pi]$ is then defined by

$$
\cos \alpha=-\sin i \sin 2 \pi \Phi,
$$

where $i \in[0, \pi / 2]$ is the orbital inclination. The flux from the planet at a phase angle $\alpha$ relative to that at $\alpha=0$ is denoted by the phase function $\phi(\alpha)$. In the case of a Lambert law sphere, the phase-dependent flux ratio $f(\Phi, i)$ is given by (Sobolev 1975)

$$
f(\Phi, i)=\epsilon \phi(\alpha)=p\left(\frac{R_{p}}{a}\right)^{2}\left[\frac{\sin \alpha+(\pi-\alpha) \cos \alpha}{\pi}\right] .
$$

For this analysis, we assume the phase variation of the reflected light is described by equation (3). The phase functions of the gas giants of our solar system are well approximated as Lambert spheres (see, e.g., Pollack et al. 1986).

In the case of $\tau$ Boo, Baliunas et al. (1997) can exclude a sinusoidal photometric variation at the planetary orbital period with a peak-to-peak amplitude of 0.4 millimag or greater. The predicted variation due to a highly reflective companion of Jupiter size is $\sim 0.1$ millimag. If proposed photometric satellite 
missions ${ }^{2}$ can achieve a precision of $\sim 10 \mu$ mag with stability over timescales of a few days, they could measure this photometric modulation, as discussed by Charbonneau (1999a).

\subsection{Spectroscopic Variations}

We assume that $\tau$ Boo has a stellar mass of $M_{s}=1.2 M_{\odot}$, based on its spectral classification as an F7 V star (Perrin et al. 1977). It has a $(B-V)$ color of 0.48 , consistent with the spectral classification. The radial velocity observations (Butler et al. 1997; G. W. Marcy 1997, private communication) provide the orbital period $(P=3.3125$ days $)$, phase $\left(T_{\Phi=0}=\right.$ $2,450,526.916 \mathrm{JD})$, eccentricity $(e=0)$, and amplitude $\left(K_{s}=\right.$ $468 \mathrm{~m} \mathrm{~s}^{-1}$ ), from which a semimajor axis of $a=0.0462 \mathrm{AU}$ and a planetary mass of $M_{p}=3.89 M_{\mathrm{Jup}} / \sin i$ are calculated. The radial velocity of the planet relative to that of the star is

$$
v_{p}(\Phi, i)=-K_{s} \frac{M_{s}+M_{p}}{M_{p}} \cos 2 \pi \Phi
$$

This has a maximum amplitude of $\left|v_{p}(\Phi, i)\right| \simeq 152 \mathrm{~km} \mathrm{~s}^{-1}$.

Thus, the spectrum of the system could contain a secondary component which varies in amplitude according to equation (3) and in Doppler shift according to equation (4). Charbonneau, Jha, \& Noyes (1998) demonstrate that the effect of the reflected light component on the line profile bisector is not far from current observational limits for a CEGP of high reflectivity. This is an alternate technique which may be used to directly detect or limit the reflected light from a CEGP.

\subsection{Tidal Locking Effects}

Baliunas et al. (1997) use the activity-rotation relation of Noyes et al. (1984) and the mean Ca II flux of $\tau$ Boo to predict a stellar rotation period of 5.1 days. Analysis of the observations of the Ca II H and K lines by Baliunas et. al (1997) yield a weakly detected period of $3.3 \pm 0.5$ days, consistent with the observed orbital period of the planet, implying that the star and planet form a tidally locked system. Marcy et al. (1997) demonstrate that, in the case of $\tau$ Boo, a convective envelope of mass $M_{\mathrm{CE}} \approx 0.01 M_{\odot}$ could become tidally locked in less than the age of the system. If so, then there is no relative motion of any point on the surface of the planet relative to any point on the surface of the star. In this case, the planet reflects a non-rotationally broadened stellar spectrum, with a typical line width dominated by the stellar photospheric convective motions ( 4 $\mathrm{km} \mathrm{s}^{-1}$; Baliunas et al. 1997). Thus, one might expect relatively narrow planetary lines superimposed on much broader stellar lines.

\section{TARGET SELECTION AND OBSERVATIONS}

Several considerations entered into the choice of $\tau$ Boo (HR 5185, HD 120136) as the optimal candidate for this experiment. First, the semimajor axis of $\tau$ Boo was smaller than that of the other three CEGPs (51 Peg, $v$ And, and $\rho^{1} \mathrm{Cnc}$ ) known at the time, which is desirable since the relative amplitude of the reflected light decreases with the square of the planet-star distance. Second, the visual brightness of $\tau$ Boo is greater than either $51 \mathrm{Peg}$ or $\rho^{1} \mathrm{Cnc}$. The photon noise of the

\footnotetext{
${ }^{2}$ See http://www.astro.ubc.ca/E-Cass/VE-98/matthews/index.html, maintained by J. M. Matthews, and http://ftp.astrsp-mrs.fr/www/arti_corot.html maintained by D. Rouan.
}

star is the dominant source of noise in the experiment, and a brighter star allows for a more precise determination of the stellar flux in a given amount of observing time. Third, as discussed above, it may be that the star is rotating with the planetary orbital period. If so, the planetary spectral features would be much sharper and deeper than those of the primary, which might facilitate their separation.

We observed $\tau$ Boo for three nights (1997 March 20-22) using the HIRES echelle spectrograph (Vogt et al. 1994) mounted on the Keck I 10 m Telescope at the W. M. Keck Observatory located atop Mauna Kea in Hawaii. These nights were carefully selected based on the phase of the companion's orbit. The spectral range used in this analysis was 465.8-498.7 $\mathrm{nm}$, and the observations were made at a resolution $R \equiv$ $\lambda / \delta \lambda$ of either 60,000 (March 20) or 45,000 (March 21 and 22).

Since the apparent magnitude of $\tau$ Boo is $4.5 \mathrm{mag}$, the high flux from the star would saturate the detector pixels for an exposure time less than the readout time of the CCD. To avoid this readout-time-limited scenario, the cross-disperser was slowly trailed during each observation so as to spread the photons over roughly 30 pixels. This allowed for typical exposure times of $300 \mathrm{~s}$, which resulted in a duty cycle of $\sim 70 \%$. In all, 154 spectra of $\tau$ Boo were obtained, with a nightly breakdown of 38 for March 20, 32 for March 21, and 84 for March 22. Cloudy weather degraded the number and quality of the spectra on March 21.

\section{DATA ANALYSIS}

\subsection{Extraction of the One-Dimensional Spectra}

Since the extraction of the one-dimensional spectra from the two-dimensional exposures must be accomplished without introducing systematic errors above the level of $1 \times 10^{-4}$ per dispersion element, it was necessary to create an entirely new and independent set of extraction codes specific to this experiment. By so doing, we were able to treat the sources of systematic noise particular to the Keck HIRES and these observations, as well as have the necessary control in identifying sources of contamination as our understanding of the data proceeded.

To extract the one-dimensional spectra from an individual frame, the following algorithm was applied: the bias was subtracted and the nonlinear gain was corrected. A twodimensional scattered light model was derived by fitting the interorder scattered light, and subtracted. The two-dimensional flat-field correction was applied, and the orders were extracted by summing along the cross-dispersion direction, making use of windows which we had produced to identify the location of both the spectral orders and the cosmetic defects from internal reflections and a felt-tip pen mark. The one-dimensional spectra were then corrected for cosmic rays by cubic spline interpolation across contaminated pixels. A low-amplitude source of high-frequency noise in the extracted spectra was corrected for by applying a narrow notch filter in Fourier space. The typical signal-to-noise ratio (SNR) was $~ 1500$ per dispersion element. The wavelength solution was derived from extracted Th and Ar emission line spectra taken throughout the observing run.

\subsection{The Model}

The model is that the data contain a secondary spectrum, identical to that of the primary, but Doppler shifted due to the 


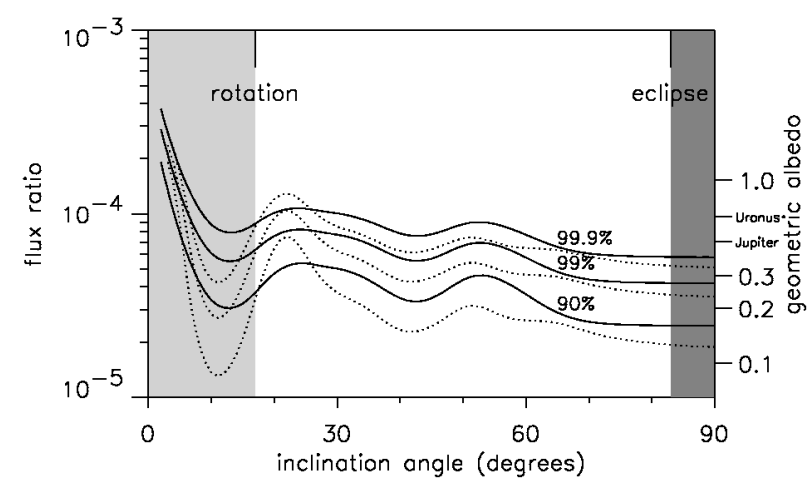

FIG. 1.-Solid curves are the 90\%, 99\%, and 99.9\% confidence levels on the upper limit for the relative reflected flux $\epsilon$ as a function of orbital inclination $i$, if the reflected light is a copy of the stellar spectrum. The dashed curves are the same confidence levels under the assumption that the system is tidally locked and thus the planet reflects a non-rotationally broadened copy of the stellar spectrum. Upper limits on the geometric albedo $p$ under the assumption that $R_{p}=1.2 R_{\mathrm{Jup}}$ are shown on the right-hand axis, and the values for Jupiter and Uranus are included for comparison. The lack of transits excludes $i \gtrsim 83^{\circ}$, and $i \lesssim 17^{\circ}$ can be excluded under the assumption that the stellar rotation axis is coaligned with that of orbital motion, as discussed in the text.

orbital motion of the planet and varying in amplitude with the angle subtended between the star, the planet, and the observer. The key to the method is first to produce a stellar template spectrum and then to make use of the orbital parameters from the radial velocity observations to calculate a model for a given observation taken at a particular orbital phase. The methods we briefly describe here will be presented in detail in an upcoming paper (Charbonneau 1999b).

The high SNR stellar template spectrum was produced for each of the two instrumental resolutions by combining all of the extracted spectra. Initially, a high SNR spectrum was chosen and an optimized model was found which corrected each observation to this reference (allowing for variations in the wavelength solution and instrumental profile, and low-frequency spatial variations of the continuum). A summed stellar template spectrum was produced, and this process was iterated twice, beyond which point the errors were no longer significantly reduced by further iteration. The errors in the summed stellar template were 1.2 times the expectation from photon noise, indicating a precision of $\sim 1 \times 10^{-4}$ per dispersion element. We note that this may well comprise the most precise visible stellar spectrum for any star other than the Sun.

For each observed spectrum, we first modify the stellar template spectrum in order to correct for the aforementioned variations in the wavelength solution and instrumental profile, and low-frequency spatial variations of the continuum. Note that we wish to interpolate the stellar template spectrum and not perform the reverse procedure and interpolate the observed spectra, since the stellar template spectrum is at a much higher SNR. Then, if we denote by $S$ this modified stellar template spectrum and by $\lambda$ the wavelength solution, the model at a given pixel $j$ of an observed spectrum taken at an orbital phase $\Phi$ is described by

$$
\begin{aligned}
& M_{j}=\left(S\left(\lambda_{j}\right)+\epsilon \phi(\Phi, i) S\left\{\lambda_{j}\left[1+\frac{v_{p}(\Phi, i)}{c}\right]\right\}\right) \\
& \quad \times[1+\epsilon \phi(\Phi, i)]^{-1} .
\end{aligned}
$$

The two unknown parameters are $\{\epsilon, i\}$. The situation in which there in no reflected light from the planet is equivalent to $\epsilon=0$. In this case, the observed spectra are best fitted as replicated stellar spectra.

As noted earlier, the stellar rotation period may be the orbital period of the planet, and hence the reflected spectrum may be composed of non-rotationally broadened lines. The instrumental resolution will smear all spectral lines to a width of $\sim 7$ $\mathrm{km} \mathrm{s}^{-1}$. Several exposures of the sharp-lined F8 V star $36 \mathrm{UMa}$ (HR 4112, HD 90839, $V=4.84, B-V=0.52$ ) were combined and corrected to the Doppler shift of $\tau$ Boo to produce a stellar template spectrum, $S^{\prime}$. The spectral differences between an F7 V and an F8 V star are insignificant for the purposes of this analysis. The spectrum of $36 \mathrm{UMa}$ serves as an excellent mock-up for the non-rotationally broadened spectrum of $\tau$ Boo and includes the instrumental effects. Thus, we also investigated the model

$$
\begin{aligned}
M_{j}^{\prime} & =\left(S\left(\lambda_{j}\right)+\epsilon \phi(\Phi, i) \gamma S^{\prime}\left\{\lambda_{j}\left[1+\frac{v_{p}(\Phi, i)}{c}\right]\right\}\right) \\
& \times[1+\epsilon \phi(\Phi, i)]^{-1},
\end{aligned}
$$

where $\gamma$ is a normalization factor.

The model was evaluated by calculating the $\chi^{2}$ parameter as a function of $\{\epsilon, i\}$. The minimum $\chi_{\min }^{2}$ is subtracted off to define $\Delta \chi^{2}=\chi^{2}-\chi_{\min }^{2}$. The confidence levels in the allowed values of the parameters are described by drawing contours of fixed $\Delta \chi^{2}$ at a desired set of significance levels. The confidence levels were tested for a given choice of $\{\epsilon, i\}$ by directly injecting a reflected light secondary at the correct amplitude and Doppler shift into each observed spectrum. At $\epsilon \gtrsim 10^{-3}$ and high inclination, the secondary can be detected at the $99 \%$ confidence level with only one spectrum. At $\epsilon \simeq 10^{-4}$, the planet is recovered only by considering all of the spectra, and the uncertainty in the parameters is significantly greater. Tests showed that the planet could be recovered for $i \gtrsim 10^{\circ}$.

A second test was provided by the detection of solar contamination employing a model similar to the one described in equation (6), but with the modification that the secondary spectrum is at a constant (but unknown) amplitude and Doppler shift. Solar contamination was detected at the Doppler shift between the Sun and $\tau$ Boo, and at a relative amplitude of $10^{-3}$, in the spectra taken on March 21. The source of this contamination was reflection of the solar spectrum off the Moon and subsequently off the clouds which were present throughout the night. The exclusion of the contaminated spectra from the reflected light analysis did not greatly reduce the statistical significance as these spectra contained only $10 \%$ of the photons of the entire data set.

\section{RESULTS AND DISCUSSION}

We find no evidence for a highly reflective planet orbiting $\tau$ Boo. For $i \geqslant 10^{\circ}$, we can constrain the reflected flux ratio $\epsilon \lessgtr 8 \times 10^{-5}$ at the $99 \%$ confidence level, under the assumptions that the reflected light spectrum is a copy of the stellar spectrum. For $i \gtrsim 70^{\circ}$, this improves to $\epsilon \leqslant 5 \times 10^{-5}$. Assuming a planetary radius of $1.2 R_{\text {Jup }}$ (Guillot et al. 1996), this limits the geometric albedo to $p \lesssim 0.3$. Figure 1 shows the precise limit of the reflected light amplitude as a function of orbital inclination. Under the assumption that the secondary reflects a non-rotationally broadened version of the stellar spec- 
trum, this limit becomes stronger for high inclinations. The particular shape of a given confidence level in Figure 1 results from the interplay of the orbital phases and statistical weights of the set of spectra. The upper limit imposed is set primarily by the last night of observing (March 22), when the planet was near a phase of $\Phi=0$. The dip down to stronger constraints on the flux ratio at an inclination $i \simeq 15^{\circ}$ results from the first night of observing (March 20) when the planet was near inferior conjunction: only if the planet is at low inclinations will it be expected to contribute a reasonable reflected light signal and hence allow us to significantly differentiate between models.

At very low inclinations $\left(i \lesssim 10^{\circ}\right)$, this experiment is not able to exclude even very bright companions due to both the lack of a significant Doppler shift between the primary and the secondary, and the lack of a phase variation in the light from the secondary. However, these low-inclination orbits may be excluded under a further consideration: if the axis defined by the stellar rotation is the same as that of the orbit of the planet, then the observed $v \sin i \simeq 15 \mathrm{~km} \mathrm{~s}^{-1}$ for the star would imply a true rotational velocity of greater than $50 \mathrm{~km} \mathrm{~s}^{-1}$ for $i \lesssim 17^{\circ}$. Such high rotational velocities are not observed (Gray 1982) for main-sequence F7 stars. High-inclination orbits can be excluded by the lack of eclipses from photometric monitoring. Baliunas et al. (1997) exclude $i \gtrsim 83^{\circ}$. This is consistent with our experiment as we find no evidence for a companion at these high inclinations.

We reiterate that the derivation of an upper limit for the geometric albedo requires the assumption of a value for the planetary radius $\left(1.2 R_{p}\right)$ and a functional form for the phase variation (a Lambert law sphere). If the actual values are significantly different than these, then the upper limit on the geometric albedo is modified as well. For example, assuming a smaller planetary radius would permit a larger albedo (see eq. [1]).

Published predictions of the albedo of CEGPs vary by many orders of magnitude and are highly sensitive to the presence of condensates in the planetary atmosphere. Burrows \& Sharp
(1999) consider cloud formation and depletion by rainout and demonstrate that $\mathrm{MgSiO}_{3}$ will be an abundant condensate at the effective temperature of $\tau$ Boo b $(\sim 1500 \mathrm{~K})$. Marley et al. (1999) calculate both cloud-free and silicate cloud atmospheres and predict $0.35 \leqq p(480 \mathrm{~nm}) \lesssim 0.55$ for an EGP with a temperature of $1000 \mathrm{~K}$, which is greater than our upper limit of $p(480 \mathrm{~nm})=0.3$. They neglect the effects of stellar insolation on the model atmosphere. Seager \& Sasselov (1998) explicitly include the stellar flux, solve the equation of radiative transfer through a model atmosphere of $\tau$ Boo $\mathrm{b}$, and predict $p(480 \mathrm{~nm}) \simeq 0.0002$. The low albedo is due in part to the absorption of photons by $\mathrm{TiO}$ in the atmosphere. However, it may be that the $\mathrm{TiO}$ forms and rains out, and thus is not an important factor. Including the presence of $\mathrm{MgSiO}_{3}$ clouds, Seager \& Sasselov predict a larger (but still very dark) albedo of $p(480 \mathrm{~nm}) \simeq 0.003$. The reflectivity of the $\mathrm{MgSiO}_{3}$ grains at a given wavelength is highly dependent on the grain size relative to the wavelength of light. Burrows \& Sharp (1999) also predict that other condensates (such as $\mathrm{Fe}$ ) may be present at these temperatures. If iron droplets are a significant condensate, the resulting albedo would be very dark due to the high absorption at optical wavelengths. Given the current uncertainty in the models, there are many reasonable model planetary atmospheres which are consistent with our upper limit.

We have achieved the current upper limit using only a limited spectral range, and data obtained when the planet was far from opposition. It is restricted by the photon noise of the data set, not by systematic errors. By expanding the spectral range and observing on several nights when the planet is near opposition, it would be possible to significantly reduce this upper limit. It may be advantageous to conduct this experiment at shorter wavelengths, since Seager \& Sasselov (1998) predict a dramatic rise in the albedo shortward of $420 \mathrm{~nm}$.

We gratefully acknowledge the NASA/Keck Time Assignment Committee for the observing time allocation. This work was supported in part by NASA grant NAG5-7505.

\section{REFERENCES}

Baliunas, S. A., Henry, G. W., Donahue, R. A., Fekel, F. C., \& Soon, W. H. 1997, ApJ, 474, L119

Burrows, A., \& Sharp, C. M. 1999, ApJ, 512, 843

Butler, R. P., Marcy, G. W., Vogt, S. S., \& Apps, K. 1998, PASP, 110, 1389

Butler, R. P., Marcy, G. W., Williams, E., Hauser, H., \& Shirts, P. 1997, ApJ, 474, L115

Charbonneau, D. 1999a, in NATO/ASI Ser., Planets Outside the Solar System: Theory and Observations, ed. J.-M. Mariotti \& D. Alloin (Dordrecht: Kluwer), 307

- 1999 b, in preparation

Charbonneau, D., Jha, S., \& Noyes, R. W. 1998, ApJ, 507, L153

Fischer, D. A., Marcy, G. W., Bulter, R. P., Vogt, S. S., \& Apps, K. 1999, PASP, 111,50

Gray, D. F. 1982, ApJ, 261, 259

Guillot, T., Burrows, A., Hubbard, W. B., Lunine, J. I., \& Saumon, D. 1996, ApJ, 459, L35
Karkoschka, E. 1994, Icarus, 111, 174

Marcy, G. W., Butler, R. P., Williams, E., Bildsten, L., Graham, J. R., Ghez, A. M., \& Jernigan, J. G. 1997, ApJ, 481, 926

Marley, M. S., Gelino, C., Stephens, D., Lunine, J. I., \& Freedman, R. 1999, ApJ, 513, 879

Mayor, M., \& Queloz, D. 1995, Nature, 378, 355

Noyes, R. W., Hartmann, L. W., Baliunas, S. L., Duncan, D. K., \& Vaughan, A. H. 1984, ApJ, 279, 763

Perrin, M.-N., Hejlesen, P. M., Cayrel de Strobel, G., \& Cayrel, R. 1977, A\&A, 54, 779

Pollack, J. B., Rages, K., Baines, K. H., Bergstralh, J. T., Wenkert, D., \& Danielson, G. E. 1986, Icarus, 65, 442

Seager, S., \& Sasselov, D. D. 1998, ApJ, 502, L157

Sobolev, V. V. 1975, Light Scattering in Planetary Atmospheres (Oxford: Pergamon)

Vogt, S. S., et al. 1994, Proc. SPIE, 2198, 362 